Pacific Journal of Mathematics

THE EXISTENCE OF TOTALLY DENSE SUBGROUPS IN LEA 


\title{
THE EXISTENCE OF TOTALLY DENSE SUBGROUPS IN LCA GROUPS
}

\author{
M. A. KHAN
}

\begin{abstract}
A proper subgroup $H$ of an LCA group $G$ is totally dense (briefly, t.d.) in $G$ if $H \cap K$ is dense in $K$ for every closed subgroup $K$ of $G$. Let $B(G)$ and $t(G)$ denote, respectively, the subgroup of compact elements of $G$ and the maximal torsion subgroup of $G$. We say that $G$ is an admissible group if $G=B(G) \neq t(G)$. An element $g \in G$ is called a metric element of $G$ if $\overline{\langle g\rangle}$, the closure of the cyclic subgroup generated by $G$, is metrizable. The following are the main results of this paper. (1) Let $G$ be an admissible LCA group such that $G_{0}$, the component of the identity in $G$, is nonmetrizable. Then $m(G)$, the set of all metric elements of $G$, is a t.d. subgroup of $G$. (2) An LCA group $G$ contains a t.d. subgroup if and only if $G$ is an admissible group. (3) A characterization of those LCA groups is given for which the maximal torsion subgroups are t.d. in the groups.
\end{abstract}

The question of the existence of proper, dense subgroups in LCA groups has received some attention in recent years (see [3], [6] and [8]). The more special case of totally dense subgroups came to our attention through a preprint of [2], in which the authors prove, among other things, several results about the existence of pseudocompact, totally dense subgroups in certain classes of compact groups. In this paper the general question of the existence of t.d. subgroups in LCA groups is considered in Theorem 2. In Theorems 1 and 3, conditions are obtained on an LCA group so that it may contain special types of t.d. subgroups. Some interesting algebraic constraints on t.d. subgroups are obtained in Proposition 1. Our basic references for facts on abelian groups and LCA groups are [4] and [5] respectively. The symbol $\approx$ denotes group isomorphism, while we use $\cong$ for topological group isomorphism. We emphasize that our t.d. subgroups are proper subgroups. We also note that an admissible LCA group is nondiscrete and infinite, and that every monothetic subgroup of such a group is either finite cyclic or infinite and compact.

We begin with three lemmas.

Lemma 1. Let $H$ be a t.d. subgroup of an LCA group $G$. Then $t(G) \subseteq H$ and $G=B(G)$. 
Proof. Let $x \in t(G)$. Then $\langle x\rangle$ is a closed, discrete subgroup of $G$. Hence $\langle x\rangle \subseteq H$ so $t(G) \subseteq H$. Next, let $y \notin B(G)$. Then $\langle y\rangle$ is an infinite, closed, discrete subgroup so $\langle y\rangle \subseteq H$. If $G \neq B(G)$, it is clear that the set of non-compact elements of $G$ would generate the group $G$, contradicting the hypothesis that $H$ is a proper subgroup. Hence, $G=B(G)$ and the proof is complete.

LeMma 2. A proper subgroup $H$ of an admissible LCA group $G$ is $t$.d. in $G$ if and only if $\overline{H \cap K}=K$ for every monothetic subgroup $K$ of $G$.

Proof. Suppose the condition is satisfied for every monothetic subgroup. Let $L$ be an arbitrary closed subgroup of $G$, and let $x \in L$. Then $\overline{\langle x\rangle}$ say, is a monothetic subgroup of $L$ so $x \in \overline{M \cap H} \subseteq \overline{L \cap H}$. Thus $\overline{L \cap H}$ Since the reverse implication is obvious, the proof is complete.

LEMma 3. Let $G$ be an infinite, compact monothetic group. (a) For every positive integer $n, n G=\{n g: g \in G\}$ is an open subgroup of $G$; in particular, every subgroup of finite index in $G$ is open. (b) For $g \in G, n g$ is a metric element of $G$ if and only if $g$ is a metric element. (c) If $H$ is a proper, dense subgroup of $G$, then $G / H$ is divisible. (d) If $G$ is totally disconnected and $H$ is a proper subgroup such that $G / H$ is divisible, then $H$ is dense in $G$.

Proof. (a) Suppose $G=\overline{\langle x\rangle}$. Since $G$ is compact, $n G$ is also compact. Now $n x \in n G$ and $\langle x\rangle$ is dense in $G$, so $n G$ must be of finite index in $G$. Hence, $n G$ is an open subgroup. Next, let $K$ be a subgroup of index $n$ in $G$. Then $n G \subseteq K$, so $K$ is open. (b) If $g$ is a metric element, then $n g$ is also a metric element because $n g \in \overline{\langle g\rangle}$. Conversely, suppose $n g$ is a metric element and let $\overline{\langle g\rangle}=H$. It is clear that $\langle n g\rangle$ is of finite index in $H$, so it is an open, metrizable subgroup of $H$. Hence, $H$ is metrizable. (c) Since $n G$ is open for every $n$, we have $G=H+n G$. Let $g+H \in G / H$ and let $m$ be a positive integer. Then $g=h_{1}+m g_{1}$ for some $h_{1} \in H, g_{1} \in G$. Now $g+H=m g_{1}+H=m\left(g_{1}+H\right)$. Hence, $G / H$ is divisible. (d) Assume $\bar{H}$ is a proper subgroup of $G$. Then $G / H$ would be totally disconnected. By our hypothesis, $G / \bar{H}$ would also be divisible and compact (hence also connected), a contradiction. Hence, $\bar{H}=G$, and the proof is complete.

We are now ready to present our first theorem, for which we draw heavily from the results in [9].

THEOREM 1. Let $G$ be an admissible LCA group such that $G_{0}$ is nonmetrizable. Then $m(G)$ is a t.d. subgroup of $G$. 
Proof. First, we show $m(G)$ is a subgroup of $G$. Let $H_{1}, H_{2}$ be compact, metrizable subgroups of $G$ and let $H=H_{1}+H_{2}$. Let $K_{1}=$ $A\left(\hat{H}, H_{i}\right)$ be the annihilator of $H_{l}$ in $\hat{H}$ for $i=1,2$. Then $K_{1} \cap K_{2}=\{0\}$ and $\hat{H} / K_{1}$ and $\hat{H} / K_{2}$ are both countable. Then $\hat{H}$ is also countable so $H$ is metrizable. Since the negative of a metric element is clearly metric, it now follows that $m(G)$ is a subgroup of $G$. Since $m\left(G_{0}\right)=m(G) \cap G_{0}$ and $G_{0}$ is nonmetrizable, it follows that $m(G)$ is a proper subgroup of $G$ [9, Theorem 3.9]. Clearly $t(G)$ is a subgroup of $m(G)$. Let $H$ be an infinite monothetic subgroup of $G$. Then $m(H)=m(G) \cap H$. By [9, Theorem 3.6], $m(H)$ is dense in $H$. Hence, $m(G)$ is t.d. in $G$ by our Lemma 2 , and the proof is complete.

COROLlaRy 1. Let $G$ be a compact, abelian group such that $G_{0}$ is nonmetrizable. Then $m(G)$ is a pseudocompact, $t$.d. subgroup of $G$.

Proof. Clearly $G$ is admissible. The assertion about pseudocompactness follows from [9, Theorem 3.6].

The next proposition brings out some interesting properties of t.d. subgroup.

Proposition 1. Let $G$ be an admissible LCA group. (a) If $H$ is t.d. in $G$, then $G / H$ is divisible. (b) $G / m(G)$ is divisible and torsion-free. (c) If $G$ is divisible, then $m(G)$ is divisible. (d) If $G$ is compact, connected and nonmetrizable, then $m(G)$ is a divisible, connected pseudocompact, t.d. subgroup of $G$.

Proof. (a) Suppose $G / H$ is not divisible, then it has a maximal subgroup [4, Exercise 1, p. 99]. So there is a t.d. subgroup $K$ containing $H$ such that $K$ is of prime index $p$. Let $X \in G, X \notin K$ and let $M=\overline{\langle x\rangle}$. Then $x$ is of infinite order and $M /(M \cap K)$ is of order $p$. But then $M \cap K$ is a proper, open subgroup of $M$, by Lemma 3(a), contradicting the fact that $\overline{M \cap K}=M$. Hence, $G / H$ must be divisible. (b) Assume $m(G)$ is a proper subgroup of $G$. By (a) above, $G / m(G)$ is divisible. Suppose $g \notin m(G)$; then $g$ is of infinite order and $n g \notin m(G)$ for any nonzero integer $n$ [Lemma 3(b)]. This means $G / H$ is torsion-free. (c) Since $G / m(G)$ is torsion-free, $m(G)$ is a pure subgroup of $G$ and so is divisible if $G$ is divisible. (d) By Theorem $1, m(G)$ is t.d. in $G$. By (c) above, $m(G)$ is divisible. By [9, Theorem 3.6] $m(G)$ is pseudocompact. By [10, Theorem 2], $m(G)$ is connected. This completes the proof. 
We now intend to show in Theorem 2 below that an LCA group has a t.d. subgroup if and only if it is admissible. First we need the following three lemmas.

Lemma 4. Suppose a totally disconnected admissible LCA group $G$ contains a proper dense subgroup $K \approx Q$, the additive group of rationals. Then $G$ is divisible and there is a subgroup $L$ of $G$, containing $K$, such that $G / L \approx Q$.

Proof. First, $G$ is divisible by [1, Theorem 1]. Let $H$ be a compact, open subgroup of $G$. Since $G$ is totally disconnected and the closure of a divisible subgroup of a compact, abelian group is divisible (hence connected), $H$ contains no nontrivial divisible subgroup. Suppose, if possible, $G$ contains a subgroup $M \approx Z\left(p^{\infty}\right) \oplus Z\left(p^{\infty}\right)$, where $Z\left(p^{\infty}\right)$ is a quasicyclic group. Then $M \cap H$ is finite, so

$$
(H+M) / H \approx M / M \cap H \approx Z\left(p^{\infty}\right) \oplus Z\left(p^{\infty}\right) .
$$

Also $G / H \approx(H+K) / H \approx K / H \cap K$, which is nontrivial and $\approx \oplus_{q} A_{q}$, where for each prime $q, A_{q}$ is either trivial or $Z\left(q^{\infty}\right)$. Since $(H+M) / H$ is a subgroup of $G / H$, this is plainly impossible. Hence $t(G)$ contains at most one copy of $Z\left(p^{\infty}\right)$ for each prime $p$. Since $G$ is divisible and nondiscrete, $G \approx \oplus_{m} Q \oplus_{q} A q$. Where $A_{q}$ is as above and $m \geq c$, the cardinality of the continuum. We now take a copy of $Q$ such that $K \cap Q=\{0\}$, and a complementary summand of $G$ containing $K$. This completes the proof.

Lemma 5. Let $G$ be an admissible LCA group. Then $G$ contains a dense subgroup $H$ such that $G / H \approx Q$.

Proof. First suppose $G$ is not totally disconnected. Then $\hat{G}$ contains a copy of $Z$, so that $T$, the multiplicative group of complex numbers of absolute value one, is a homomorphic image of $G$. Let $f: G \rightarrow T$ be an open, continuous epimorphism. It is obvious that $T$ contains a dense subgroup, say $K$, such that $T / K \approx Q$. Then $H=f^{-1}(K)$ is a proper dense subgroup of $G$ with $G / H \approx Q$. Now suppose $G$ is totally disconnected. Let $E(G)$ denote the minimal divisible extension of $G$ topologized in the usual manner so that $G$ is an open subgroup. Since $G$ is not a torsion group, $E(G)$ contains a copy of $Q$, say $M$, with some nondiscrete topology. Now $\bar{M}$ is divisible [1, Theorem 1], so $E(G)=\bar{M}+L$, where $\bar{M} \cap L=\{0\}$. By Lemma $4, \bar{M}$ contains a dense subgroup $N$ such that $M \subseteq N$ and $\bar{M} / N \approx Q$. We have $E(G)=\bar{N}+L$. Since $N+L$ is a proper 
dense subgroup of $E(G),(N+L) \cap G$ is a proper dense subgroup of $G$, and

$$
\begin{aligned}
G /(N+L) \cap G & \approx(N+L+G) /(N+L)=E(G) /(N+L) \\
& =(\bar{N}+L) /(N+L) \approx \bar{N} / N \approx Q .
\end{aligned}
$$

The proof is complete.

LEMMA 6. Let $G$ be an infinite, compact, monothetic group with a subgroup $H$ such that $G / H$ is a torsion-free group of rank one. The $\bar{H}=G$ and $G / H \approx Q$.

Proof. First let $G$ be totally disconnected. By [5, 25.13] and duality, every closed subgroup of $G$ is monothetic. Now $\bar{H} / H$ is trivial or $\bar{H} / H$ is divisible, by Lemma 3 (c). So as a subgroup of $G / H, \bar{H} / H$ is trivial or $\approx$ $Q$. In the first case, $H$ would be a closed subgroup of countably infinite index in the compact group $G$, which is impossible. Hence, $\bar{H}=G$ and $G / H \approx Q$. Next, let $G$ be connected. The hypothesis ensures that $G / H \approx$ $Q$. Again, $\bar{H} \neq G$, would imply $G$ has a closed subgroup of countably infinite index. Hence, $\bar{H}=G$. Finally assume $G_{0}$ is nontrivial and proper. If $G_{0} \subseteq H$, then $\left(G / G_{0}\right) /\left(H / G_{0}\right)$ is torsion-free or rank one, so that the first case is applicable and we have that $H / G_{0}$ is dense in $G / G_{0}$ and $\left(G / G_{0}\right) /\left(H / G_{0}\right) \approx Q$. It follows, via the natural open, continuous homomorphism of $G$ onto $G / G_{0}$, that $\bar{H}=G$ and $G / H \approx Q$. If $G_{0}$ is not contained in $H$, then $G_{0} /\left(H \cap G_{0}\right) \approx\left(G_{0}+H\right) / H \subseteq G / H$. As $G_{0}$ is divisible, this implies $G_{0} /\left(G_{0} \cap H\right) \approx Q \approx G / H$, so $H+G_{0}=G$. Clearly, $\overline{\bar{G}_{0} \cap H}=G_{0}$. Hence,

$$
G=H+G_{0} \subseteq \bar{H}+\overline{G_{0} \cap H} \subseteq \bar{H},
$$

and the proof is complete.

We now come to our main result.

THEOREM 2. An LCA group $G$ contains a t.d. subgroup if and only if $G$ is an admissible group.

Proof. If $G$ has a t.d. subgroup, Lemma 1 ensures that $G$ is admissible. Conversely, $G$ contains a dense subgroup $H$ such that $G / H \approx Q$ [Lemma 5]. Let $K$ be an arbitrary compact, monothetic subgroup of $G$. If $K$ is finite, then clearly $K \subseteq H$. Assume $K$ is infinite. Then

$$
(K+H) / H \approx K /(K \cap H) \approx \text { a subgroup of } Q .
$$


So $K /(K \cap H)$ is either trivial or $\approx$ to a torsion-free subgroup of rank one. In the first case, $K \subseteq H$; in the second, $\overline{K \cap H}=K$ by Lemma 6 . An appeal to Lemma 2 completes the proof.

COROllary 2. A compact abelian group $G$ contains a $t$.d. subgroup if and only if $G$ is not a torsion group.

Proof. A compact abelian group is admissible exactly when it is not a torsion group.

COROllaRY 3. Let $G$ be an admissible LCA group. Then $G$ contains a t.d. subgroup $H$ such that $G / H \approx Q$. Moreover, every dense subgroup $L$ satisfying $G / L \approx Q$ is $t$.d. in $G$.

Proof. A perusal of the proof of Theorem 2 leads immediately to a proof of this corollary.

We now deal with the question: When is $t(G)$ a t.d. subgroup of an LCA group $G$ ? First we prove a lemma.

LEMMA 7. Let $G$ be an infinite, compact abelian group containing no nontrivial closed torsion-free subgroup. Then $t(G)$ is dense in $G$.

Proof. By [5, 24.24] $t(G)$ is dense in $G$ if and only if $\cap_{n=1}^{\infty} n \hat{G}$ is trivial. Combining Proposition 4 with Lemma 1 of [7], we get that $t(G)$ is dense in $G$.

Just as Theorem 1 depends on the results in [9], our next theorem depends on the results in [7]. We recall that an LCA group $G$ has property $C$ if every proper closed subgroup of $G$ is contained in a maximal (proper) subgroup of $G$. Several characterizations of property $C$ appear in Theorem 3 of [7]. We also recall that an abelian group $G$ is called totally reduced if every nontrivial homomorphic image of $G$ is reduced. (For various results concerning totally reduced abelian groups, see [7, Propositions 1-4]).

THEOREM 3. An LCA group $G$ has $t(G)$ as a t.d. subgroup if and only if $G$ is not a torsion group and $\hat{G}$ has property $C$.

Proof. Suppose $t(G)$ is t.d. in $G$. Then $G$ is not a torsion group. Also $G$ contains no nontrivial closed torsion-free subgroup. Hence, $\hat{G}$ has property $C$ [7, Theorem 3]. For the converse, we note first that $\hat{G}$ is totally disconnected [7, Theorem 3(b)], so duality yields $G=B(G)$. Thus $G$ is an 
admissible group. Let $K$ be an infinite, compact subgroup of $G$. Then by Lemma 7, $t(G) \cap K$ is dense in $K$. By Lemma 2 we conclude that $t(G)$ is t.d. in $G$, and the proof is complete.

Corollary 1. A compact, abelian group $G$ has $t(G)$ as a t.d. subgroup if and only if $\hat{G}$ is totally reduced and not of bounded order.

Proof. Since $\hat{G}$ is a discrete group, property $C$ is the same as the property of being totally reduced. Again, the compact group $G$ is not a torsion group if and only if the orders of the elements of $\hat{G}$ are not bounded.

Finally, we set down a few results in the next proposition to provide some more feeling for compact, abelian groups with totally dense torsion subgroups.

Proposition 2. Let $G$ be a compact, abelian group with $t(G)$ as a t.d. subgroup. Then the following hold:

(a) $G$ is finite dimensional.

(b) If $G$ is connected, then $G$ is metrizable and monothetic.

(c) If $G$ is monothetic, then $G$ is metrizable and $G / G_{0} \cong$ topological direct product $\Pi_{p} A_{p}$, where $A_{p}$ is trivial or a discrete cyclic p-group for every prime $p$.

Proof. (a) $\hat{G}$ is totally reduced. Also $r(\hat{G})=r(\hat{G} / t(\hat{G}))$, where $r(\hat{G})$ denotes the torsion-free rank of $\hat{G}$ (see [4, Exercise 3, p. 86]). By [7, Proposition 3(b)], $r(\hat{G} / t(\hat{G}))$ is finite. Hence, $G$ is finite dimensional [5, 24.28].

(b) If $G$ is connected also, then $\hat{G}$ is torsion-free and totally reduced. Here $\hat{G}$ is isomorphic with a torsion-free, countable subgroup of $T_{d}$, and so $G$ is metrizable and monothetic [5, Theorems 24.15 and 24.32].

(c) In this case $\hat{G}$ is a totally reduced subgroup of $T_{d}$. From [7, Proposition 3] and the structure of $T_{d}$, is is clear that $G$ is countable and the $p$-rank of $t(\hat{G})$ is 0 or 1 for every $p$. By duality the stated result follows.

\section{REFERENCES}

[1] D. L. Armacost and W. L. Armacost, On Q-dense and densely divisible LCA groups, Proc. Amer. Math. Soc., 36 (1972), 301-305.

[2] W. W. Comfort and T. Soundararajan, Pseudocompact topological group topologies, Pacific J. Math., (to appear). 
[3] W. E. Dietrich, Jr., Dense decompositions of locally compact groups, Colloq. Math., 24 (1972), 147-151.

[4] L. Fuchs, Infinite Abelian Groups, Vol. 1, Academic Press, New York, 1970.

[5] E. Hewitt and K. Ross, Abstract Harmonic Analysis, Vol. 1, Academic Press, New York, 1963.

[6] M. A. Khan, Chain conditions on subgroups of LCA groups, Pacific J. Math., 86 (1980), 517-534.

[7] When are proper subgroups of LCA groups contained in maximal ones?, Proc. Amer. Math. Soc., 83 (1981), 131-137.

[8] M. Rajagopalan and H. Subrahmanian, Dense subgroups of locally compact groups, Colloq. Math., 35 (1976), 289-292.

[9] H. J. Wilcox, Pseudocompact groups, Pacific J. Math., 19 (1966), 365-379.

[10] _ Dense subgroups of compact groups, Proc. Amer. Math. Soc., 28 (1971), $578-580$.

Received August 4, 1982.

1-C, 5/13, NAZIMABad

Karachi, Pakistan 


\title{
PACIFIC JOURNAL OF MATHEMATICS EDITORS
}

\author{
Donald BabBITT (Managing Editor) \\ University of California \\ Los Angeles, CA 90024 \\ Hugo Rossi \\ University of Utah \\ Salt Lake City, UT 84112 \\ C. C. Moore and Arthur Ogus \\ University of California \\ Berkeley, CA 94720
}

\author{
J. Dugundu \\ Department of Mathematics \\ University of Southern California \\ Los Angeles, CA 90089-1113
}

R. FINN and H. SAMELSON

Stanford University

Stanford, CA 94305

\section{ASSOCIATE EDITORS}
R. ARENS
E. F. BECKENBACH
B. H. NeumanN
F. WolF
K. YosHIDA (1906-1982)

\section{SUPPORTING INSTITUTIONS}

\author{
UNIVERSITY OF ARIZONA \\ UNIVERSITY OF BRITISH COLUMBIA \\ CALIFORNIA INSTITUTE OF TECHNOLOGY \\ UNIVERSITY OF CALIFORNIA \\ MONTANA STATE UNIVERSITY \\ UNIVERSITY OF NEVADA, RENO \\ NEW MEXICO STATE UNIVERSITY \\ OREGON STATE UNIVERSITY
}

\author{
UNIVERSITY OF OREGON \\ UNIVERSITY OF SOUTHERN CALIFORNIA \\ STANFORD UNIVERSITY \\ UNIVERSITY OF HAWAII \\ UNIVERSITY OF TOKYO \\ UNIVERSITY OF UTAH \\ WASHINGTON STATE UNIVERSITY \\ UNIVERSITY OF WASHINGTON
}

The Supporting Institutions listed above contribute to the cost of publication of this Journal, but they are not owners or publishers and have no responsibility for its content or policies.

Mathematical papers intended for publication in the Pacific Journal of Mathematics should be in typed form or offset-reproduced (not dittoed), double spaced with large margins. Please do not use built up fractions in the text of the manuscript. However, you may use them in the displayed equations. Underline Greek letters in red, German in green, and script in blue. The first paragraph must be capable of being used separately as a synopsis of the entire paper. In particular it should contain no bibliographic references. Please propose a heading for the odd numbered pages of less than 35 characters. Manuscripts, in triplicate, may be sent to any one of the editors. Please classify according to the scheme of Math. Reviews, Index to Vol. 39. Supply name and address of author to whom proofs should be sent. All other communications should be addressed to the managing editor, or Elaine Barth, University of California, Los Angeles, California 90024.

There are page-charges associated with articles appearing in the Pacific Journal of Mathematics. These charges are expected to be paid by the author's University, Government Agency or Company. If the author or authors do not have access to such Institutional support these charges are waived. Single authors will receive 50 free reprints; joint authors will receive a total of 100 free reprints. Additional copies may be obtained at cost in multiples of 50 .

The Pacific Journal of Mathematics is issued monthly as of January 1966. Regular subscription rate: $\$ 132.00$ a year (6 Vol., 12 issues). Special rate: $\$ 66.00$ a year to individual members of supporting institutions.

Subscriptions, orders for numbers issued in the last three calendar years, and changes of address should be sent to Pacific Journal of Mathematics, P.O. Box 969, Carmel Valley, CA 93924, U.S.A. Old back numbers obtainable from Kraus Periodicals Co., Route 100, Millwood, NY 10546.

The Pacific Journal of Mathematics ISSN 0030-8730 is published monthly by the Pacific Journal of Mathematics at P.O. Box 969, Carmel Valley, CA 93924. Application to mail at Second-class postage rates is pending at Carmel Valley, California, and additional mailing offices. Postmaster: Send address changes to Pacific Journal of Mathematics, P. O. Box 969, Carmel Valley, CA 93924.

PUBLISHED BY PACIFIC JOURNAL OF MATHEMATICS. A NON-PROFIT CORPORATION

Copyright $\odot 1984$ by Pacific Journal of Mathematics 


\section{Pacific Journal of Mathematics}

\section{Vol. 112, No. $2 \quad$ February, 1984}

Kenneth F. Andersen and Wo-Sang Young, On the reverse weak type inequality for the Hardy maximal function and the weighted classes

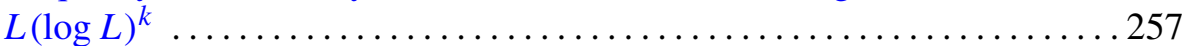

Richard Eugene Bedient, Double branched covers and pretzel knots ..... 265

Harold Philip Boas, Holomorphic reproducing kernels in Reinhardt

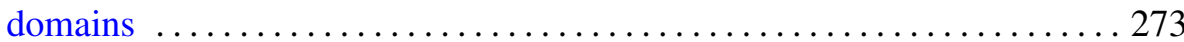

Janey Antonio Daccach and Arthur Gabriel Wasserman, Stiefel's theorem and toral actions ................................. 293

Michael Fried, The nonregular analogue of Tchebotarev's theorem ....... 303

Stanley Joseph Gurak, Minimal polynomials for circular numbers . . . . . . 313

Norimichi Hirano and Wataru Takahashi, Nonlinear ergodic theorems for an amenable semigroup of nonexpansive mappings in a Banach space . . 333

Jim Hoste, Sewn-up $r$-link exteriors . . . . . . . . . . . . . . . . . . . 347

Mohammad Ahmad Khan, The existence of totally dense subgroups in

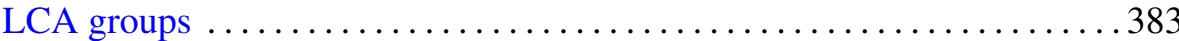

Mieczysław Kula, Murray Angus Marshall and Andrzej Sładek, Direct

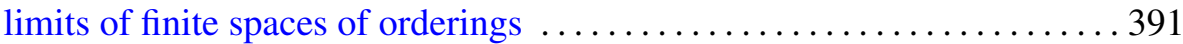

Luis Montejano Peimbert, Flat Hilbert cube manifold pairs ........... 407

Steven C. Pinault, An a priori estimate in the calculus of variations . . . . . 427

McKenzie Y. K. Wang, Some remarks on the calculation of Stiefel-Whitney classes and a paper of Wu-Yi Hsiang's

Brian Donald Wick, The calculation of an invariant for Tor . . 445

Wolfgang Wollny, Contributions to Hilbert's eighteenth problem 